\section{Matrilysine 1 et pathologie cancéreuse}

Lionel Rémy, Cécile Trespeuch
> La matrilysine 1 (matrix metalloproteinase 7, MMP7) a une importance considérable dans la progression des cancers, notamment digestifs, non seulement parce qu'elle exerce un rôle direct de dégradation sur des molécules cibles à différents niveaux, mais aussi parce qu'elle intervient dans l'activation d'autres systèmes protéolytiques. Son expression, faible dans les tissus sains, augmente très nettement au cours des proliférations cellulaires bénignes de type adénome, pour croître encore dans les cancers et être impliquée aussi bien au niveau du développement tumoral qu'à celui de la prolifération métastatique. Elle agit aux différentes étapes de la transformation maligne en clivant la $\varepsilon$-cadhérine, molécule d'adhérence intercellulaire des épithéliums, ainsi que les molécules matricielles à l'interface tumeurstroma et les intégrines qui les unissent aux cellules épithéliales. L'utilisation d'oligonucléotides antisens pour la MMP7 entraîne une nette diminution des processus invasifs, suggérant ainsi un potentiel thérapeutique important. <

La matrilysine 1 (MMP7) a été identifiée à l'origine dans I'utérus de rat in vivo [1] et dans le milieu de culture de la lignée de carcinome rectal humain CaR-1 [2]. Elle fait partie de la famille des métalloprotéases, enzymes protéolytiques formées de cinq domaines de base: un prédomaine ou séquence signal, un prodomaine ou domaine de latence, un domaine catalytique lié au zinc, une région charnière et un domaine dont la séquence est homologue à celle de l'hémopexine, une protéine plasmatique, et la vitronectine, une protéine d'adhérence. La MMP7 ne présente que les trois premiers domaines, requis pour la sécrétion et l'activité. Cette structure particulière pourrait soit résulter de son évolution à partir de structures ancestrales, soit représenter une forme primitive des métalloprotéases qui se constitueraient alors par ajout d'exons.

Article reçu le 25 octobre 2004 et accepté le 9 février 2005 .

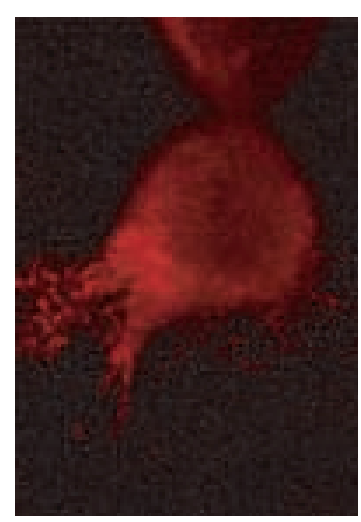

Rôles de MMP7 dans la carcinogenèse
Inserm U.45, IFR62, Université Lyon 1, Faculté de Médecine Laennec, 7, rue Guillaume Paradin, 69372 Lyon Cedex 08, France. remy@lyon.inserm.fr
Présente dans certains tissus normaux à caractère glandulaire exocrine comme la glande mammaire, la prostate, le pancréas, le foie ou la glande parotide, elle intervient dans la réparation tissulaire et la différenciation osseuse, mais est surtout remarquable pour sa présence ubiquitaire dans divers cancers, notamment digestifs. Son expression, qui est retrouvée dans l'adénome comme dans le carcinome du côlon, concerne aussi l'estomac et les voies aérodigestives supérieures. Elle est impliquée dans la formation des tumeurs et dans la dégradation tissulaire consécutive à l'extravasation des cellules tumorales. Contrairement aux autres métalloprotéases, principalement d'origine stromale [3], elle est synthétisée et exportée par les cellules épithéliales. Si son ARNm est observé dans les tumeurs dès le stade de l'adénome avec dysplasie sévère, son niveau d'expression reste inférieur à ce qu'il est dans les cancers. Des souris Min (multiple intestinal neoplasia), dont $88 \%$ des adénomes expriment l'ARNm de la MMP7, montrent une diminution de $60 \%$ des adénomes, en nombre comme en volume, quand elles sont rendues déficientes pour le gène codant pour la MMP7 par recombinaison homologue, en corrélation avec une diminution drastique de la protéine [4]. L'action de la MMP7 s'expliquerait, dans ce cas précis, par le fait que la protéine étant détectée à la face luminale des glandes adénomateuses, elle activerait des cytokines 
liées aux membranes ou des facteurs de croissance, contribuant ainsi au développement des tumeurs bénignes.

\section{MMP7 et invasion}

Le rôle de la MMP7 a principalement été étudié dans le cadre plus général du processus invasif, comme le soulignent les exemples suivants. En cas de rectocolite ulcéreuse, les cellules épithéliales jouxtant l'ulcération expriment nettement la MMP7, de façon focale ou diffuse en fonction du niveau de la dysplasie [5], et avec une augmentation en relation avec la sévérité de l'inflammation, ce qui pourrait conférer un caractère prédictif de transformation chez les patients souffrant de cette maladie [6].

Par ailleurs, il a été montré que la surexpression de la MMP7 par une lignée de cancer de la prostate normalement peu invasive multipliait par 6 son invasivité, suggérant un rôle fonctionnel de la protéase au stade initial de l'invasion [3]. Dans le cancer gastrique, le niveau d'expression est 5 à 40 fois supérieur à celui des MMP1, 2, 3 et 9 , toutes impliquées dans la progression tumorale [7]. Dans une étude sur l'œsophage de Barrett, les auteurs ont montré que la MMP7 était exprimée en abondance dans 12 tumeurs primitives sur 15, ainsi que dans 4 métastases ganglionnaires sur 6 [8]. Principale métalloprotéase détectée avec la MMP9 [9], elle est exprimée non seulement au niveau du front d'invasion, mais par l'ensemble de la tumeur. Son ARNm est détecté dès le stade métaplasie, ce qui montre une régulation précoce au cours du phénomène de l'oncogenèse. Localisée à l'interface de la tumeur avec le stroma dans environ un cas sur deux, son expression est significativement $(p=0,004)$ corrélée avec la profondeur de l'invasion, mais aussi avec l'envahissement ganglionnaire [8], le stade d'avancement et la récidive dans la première année qui suit le traitement chirurgical. Son identification se révèle alors utile au pronostic et à la prédiction des rechutes [10]. Dans les tumeurs colorectales, le taux d'ARNm de la MMP7 augmente

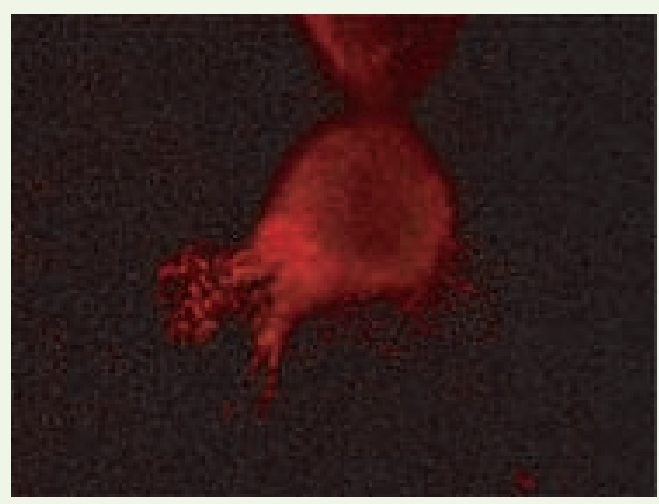

Figure 1. Concentration de la MMP7 (matrix metalloproteinase 7) à l'extrémité d'un lamellipode de cellule de carcinome de côlon humain Isreco-1 en culture. avec le stade d'avancement, montrant une expression particulièrement forte dans les métastases hépatiques [11]. La présence de MMP7 est également significativement corrélée avec l'invasion des voies lymphatiques et l'existence de métastases ganglionnaires. Sa localisation au front de la tumeur prédit un pronostic défavorable, les cellules pouvant alors exercer leur action protéolytique sur les molécules matricielles directement à leur contact, à l'interface de la tumeur et du stroma [12]. Par ailleurs, la MMP7 induit une agrégation des cellules tumorales en s'accrochant à leur surface, formant alors des colonies cellulaires qui vont s'implanter dans les sites métastatiques [13]. La polarisation de la MMP7 se retrouve dans les systèmes in vitro: nous avons ainsi montré une concentration de la protéase à l'extrémité des lamellipodes des cellules cancéreuses coliques humaines Isreco-1 [14] (Figure 1). Ce résultat rappelle ce qui est observé dans des cultures de cellules gastriques infectées par Helicobacter pylori, qui montrent une augmentation de l'expression de la MMP7 au front de migration des colonies cellulaires, préférentiellement au niveau des lamellipodes, ainsi qu'une vitesse d'étalement accrue par rapport à celle des cellules non infectées, ce phénomène étant inhibé par des antisens de la MMP7 [15]. Ces observations mettent clairement en évidence le lien existant entre la propension des cellules tumorales à produire des expansions cytoplasmiques et une régulation positive de la MMP7.

\section{MMP7 et angiogenèse}

Au-delà de l'invasion localisée, le support de la dissémination métastatique repose sur l'angiogenèse tumorale, phénomène dans lequel la MMP7 joue un rôle très important. Les cellules endothéliales des vaisseaux adjacents aux tumeurs exprimant la MMP7 I'expriment elles-mêmes, ainsi que son ARNm, à la différence des cellules endothéliales adjacentes à des tumeurs ne l'exprimant pas. Les cellules endothéliales et tumorales seraient donc régulées de façon paracrine par des facteurs solubles communs, favorisant l'angiogenèse tumorale et donc l'envahissement métastatique [16]. Des travaux plus récents du même groupe ont montré que la culture de cellules endothéliales de veine ombilicale humaine (HUVEC) sur du collagène I ou IV en présence de MMP7 recombinante provoque une prolifération dose-dépendante de ces cellules [17]. La MMP7 ne faisant pas croître le taux de VEGF (vascular endothelial growth factor), on peut supposer que son action sur l'angiogenèse est directe, sans toutefois exclure une possible prolifération des HUVEC sous l'action d'autres métalloprotéases (MMP1, 2 et 9), dont les formes latentes sont activées par la MMP7 recombinante. À ce jour, le mécanisme demeure inconnu. D’un autre côté, la MMP7 produit in vivo de l'angiostatine à partir du plasminogène circulant, inhibant ainsi la prolifération des cellules endothéliales, et ce sous le contrôle de l'intégrine $\alpha 1 \beta 1$. En effet, des souris invalidées $\alpha \mathrm{l} \beta \mathrm{l}$ null montrent une nette augmentation de la synthèse d'angiostatine [18]. L'effet de la 
MMP7 sur l'angiogenèse est donc complexe: si elle fait proliférer les vaisseaux in vitro, son effet peut être opposé in vivo, lorsque la possibilité d'accroître la synthèse d'angiostatine lui est donnée.

\section{MMP7 et apoptose}

Les cellules exprimant la MMP7 sont moins sensibles que les autres à l'apoptose. L'expression de la protéase à des stades très précoces de la transformation tumorale permettrait une sélection de cellules ayant une sensibilité très réduite à la destruction par le système de surveillance immunitaire, avec la possibilité d'acquérir des modifications génétiques additionnelles leur permettant de proliférer en tumeurs [19].

\section{Cibles de la MMP7}

Comme toute protéase, la MMP7 exerce sur ses cibles des actions généralement dépendantes de facteurs externes. Sa participation à la progression tumorale revêt deux aspects essentiels: une action directe sur les systèmes d'adhérence de la tumeur, aussi bien au niveau intercellulaire qu'à l'interface avec le stroma (dans ce dernier cas, elle clive les composants matriciels et certains récepteurs à l'origine de l'adhérence des cellules sur ces composants), et une action indirecte, en modulant l'action d'autres protéases.

Ces actions sont influencées par des facteurs externes, dont certains sont essentiels puisque la MMP7, synthétisée sous une forme immature (28 kDa), ne peut agir que sous sa forme activée (19 kDa). D'autres, plus optionnels, accélèrent ou freinent sa production. La description des cibles moléculaires de la MMP7 et des facteurs régulant son action permet de la positionner dans la nébuleuse protéolytique de la maladie cancéreuse (Figure 2).

\section{Dégradation de la matrice extracellulaire}

L'un des mécanismes les plus précoces conduisant à l'invasion dans les carcinomes est la perte des contacts intercellulaires. La MMP7 clive la $\varepsilon$-cadhérine [20], principale molécule responsable de l'adhérence intercellulaire, au niveau de son ectodomaine, isolant un fragment soluble de $80 \mathrm{kDa}$. Ce clivage provoque la dissociation des cellules en culture et inhibe de façon paracrine l'agrégation cellulaire et l'invasion du collagène I. Au cours de la réparation tissulaire, le clivage de l'ectodomaine de la $\varepsilon$-cadhérine par la MMP7 favorise la migration des cellules engagées dans un processus de cicatrisation [21]. Au niveau stromal, les principales cibles de la MMP7 sont les différents composants de la matrice extracellulaire et de la membrane basale des épithéliums et des endothéliums. Elle dégrade notamment la fibronectine, le collagène de type IV, la laminine, l'élastine, l'entactine, la ténascine C, la vitronectine et les protéoglycanes [22]. D'une efficacité remarquable, elle clive l'entactine, protéine liant la laminine au collagène IV,
600 fois plus rapidement que ne le ferait la MMP9. Elle clive également certains récepteurs intégrines liant les cellules épithéliales aux composants matriciels. Ayant observé dans le carcinome prostatique une forte expression de la MMP7 corrélée à une absence de la sous-unité d'intégrine $\beta 4$, des auteurs ont montré in vitro que la sous-unité $\beta 4$ était clivée, isolant un fragment de 90 kDa [23]. Cela n'est pas sans conséquence sur l'adhérence des cellules à certains composants de la membrane basale, notamment à la laminine 5 . Ces interactions de la MMP7 avec les divers composants matriciels ont un effet promoteur sur la migration. Ainsi, une colocalisation de la MMP7 avec la chaîne $\gamma 2$ de la laminine 5 au front de tumeurs coliques a été observée [12]. Cela ne préjuge toutefois pas d'un clivage de la laminine 5 à ce niveau: en effet, des travaux en cours au sein de notre équipe tendent à montrer qu'un tel clivage concernerait plutôt la chaîne $\beta 3$ dont l'association en dimère avec $\gamma 2$ pourrait alors expliquer les observations précédentes.

\section{Activation d'autres systèmes protéolytiques}

En plus de ses effets directs sur la dégradation de la matrice extracellulaire, la MMP7 active la pro-MMP2, produite par $81 \%$ des adénocarcinomes de l'œsophage, et potentialise fortement l'action de I'APMA (4-aminophenylmercuric-acetate) dans ce processus, en quintuplant le niveau d'activité de la MMP2 obtenu avec I'APMA seul [24]. Elle active également les pro-MMPl et 9 [17].

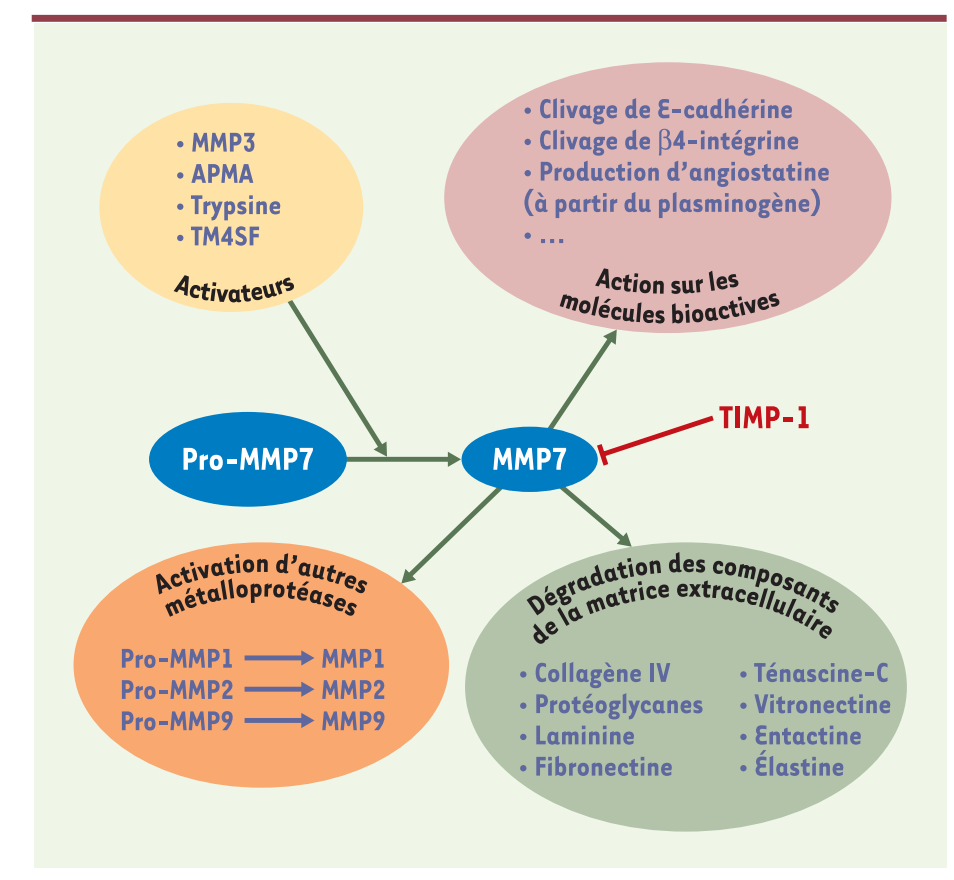

Figure 2. La MMP7 (matrix metalloproteinase 7) et ses interactions. MMP: matrix metalloproteinase; APMA: 4-aminophenylmercuric-acetate; TM4SF: transmembrane 4 superfamily. 


\section{Régulation de la MMP7}

L'expression de la MMP7 dépend de l'environnement tumoral. Ainsi, la culture de cellules d'adénocarcinome squameux de la langue en présence de fibroblastes de prépuce multiplie par 40 le taux de pro-MMP7 exprimé par les cellules cultivées seules. L'induction de pro-MMP7 est due aux interactions intercellulaires, avec mise en jeu des $N$ - et $\varepsilon$-cadhérines, ainsi qu'aux interactions entre les cellules et la matrice extracellulaire par le biais des intégrines. Le fait de neutraliser ces deux facteurs ramène la pro-MMP7 à son niveau basal, tandis qu'en neutraliser un seul réduit partiellement l'expression de la MMP7 mature dans la coculture [22].

\section{Activation}

Au cours des stades précoces de la tumorigenèse colorectale, la MMP7 est régulée positivement via la voie de signalisation de la $\beta$-caténine [25]. La mutation du gène APC (adenomatous polyposis coli), dont la conséquence est une surexpression du complexe $\beta$-caténine/TCF4, facteur de transcription pour les gènes promoteurs de la protéase, entraîne une augmentation de l'expression de la MMP7 dans $80 \%$ des cancers [26]. Cette surexpression est particulièrement marquée au front d'invasion des tumeurs où les cellules expriment fortement la $\beta$-caténine dans leur noyau et la MMP7 dans leur cytoplasme [27]. Par ailleurs, l'oncoprotéine c-jun, composant du facteur de transcription AP-1 (activating protein 1), est elle-même un activateur fort de la transcription de la MMP7 [28], tout comme le facteur de transcription Ets-1, notamment dans le carcinome hépatocellulaire [29]. Les facteurs de croissance ont également une action importante sur l'expression de la pro-matrilysine: c'est le cas du FGF-l (fibroblast growth factor 1 ), dont la signalisation passe par la ERK/MAP-kinase (extracellular signal-regulated kinase/mitogen-activated protein kinase) et le facteur de transcription STAT3 (signal transducer and activator of transcription 3) [30], et de l'EGF (epidermal growth factor), dont la signalisation passe par le facteur de transcription PEA3 (polyoma enhancer activator 3) [31]. L'action de la MMP7 ne peut se faire que sous sa forme mature. Différents activateurs de la pro-MMP7 $(28 \mathrm{kDa})$ ont été répertoriés: l'APMA permet l'obtention d'une forme intermédiaire de $21 \mathrm{kDa}$ et d'une forme active de $19 \mathrm{kDa}$. La trypsine active également entièrement la pro-MMP7 [32], tandis que la plasmine et l'élastase leucocytaire ne l'activent que partiellement [33]. Par ailleurs, le TM4SF (transmembrane 4 superfamily) capture la pro-MMP7 sur la surface des cellules carcinomateuses, où elle s'attache via des heparan sulfate proteoglycans [34] et est activée [35]. La présence de MMP3 dans les macrophages et les cellules stromales à proximité immédiate des cellules tumorales suggère une interaction privilégiée avec la MMP7, conduisant probablement à son activation [2].

\section{Inhibition}

L'inhibition de la MMP7, qui dépend préférentiellement du TIMPl (tissue inhibitor of metalloproteinase 1) [8], a un effet négatif important sur l'invasion, en raison d'une inhibition de la protéolyse directement due à la MMP7 et de celle relevant des autres MMP qu'elle régule. Toutefois, et à la différence des autres MMP, la MMP7 ne possède pas de domaine carboxyterminal, et aura donc moins d'affinité pour son inhibiteur physiologique: il en résulte probablement une plus grande résistance à l'inhibition [22], ce qui peut expliquer son efficacité particulière dans la protéolyse matricielle.

\section{Conclusions}

De par la diversité de ses cibles, la MMP7 se trouve au centre et aux commandes d'un vaste système ayant pour finalité globale de favoriser la migration et l'invasion. Toute action qu'elle subira aura de ce fait un retentissement particulièrement important sur des systèmes de protéolyse à la fois indépendants et complémentaires, dont le dénominateur commun reste la progression tumorale. Cela souligne l'intérêt d'appliquer à la MMP7 des stratégies antisens et de la soumettre à l'action d'inhibiteurs spécifiques. $\diamond$

\section{SUMMARY}

Matrilysin- 1 and cancer pathology

Matrilysin-1 has a considerable importance in the progression of cancers, namely digestive, ones inasmuch as it fulfils a direct function of deterioration on molecular targets at different levels and interfears as well in the activation of other proteolytic systems. Its low expression in sound tissues increases distinctly in mild cellular proliferations like adenomas to increase even more in carcinomas and be involved at the level of tumoral development as well as in a metastatical proliferation. It acts during the different stages of malignant transformation, splitting the $\varepsilon$-cadherin, an intercellular adhesive molecule of epithelia, matrix molecules in the tumor-stroma interface and integrins which link matrix components to epithelial cells. The use of oligonucleotide antisens to the matrilysin-1 drives to a clear reduction of invading process, suggesting therefore an important therapeutic potential. $\diamond$

\section{RÉFÉRENCES}

1. Woessner JF, Taplin CJ. Purification and properties of a small latent matrix metalloproteinase of the rat uterus. J Biol Chem 1998; 263: 16918-25.

2. Imai K, Yokohama Y, Nakanishi I, et al. Matrix metalloproteinase 7 (matrilysin) from human rectal carcinoma cells. Activation of the precursor interaction with other matrix metalloproteinases and enzymic properties. J Biol Chem 1995; 270: 6691-7.

3. Powell WC, Domann FE Jr, Mitchen JM, et al. Matrilysin expression in the involuting ventral prostate. Prostate 1996; 29: 159-68.

4. Wilson CL, Heppner KJ, Labosky PA, et al. Intestinal tumorigenesis is suppressed in mice lacking the metalloprotease matrilysin. Proc Natl Acad Sci USA 1997; $94: 1402-7$. 
5. Newell KJ, Matrisian LM, Driman DK. Matrilysin (matrix metalloproteinase-7) expression in ulcerative colitis-related tumorigenesis. Mol Carcinol 2002; 34: 59-63.

6. Matsuno K, Adachi Y, Yamamoto $\mathrm{H}$, et al. The expression of matrix metalloproteinase matrilysin indicates the degree of inflammation in ulcerative colitis. J Gastroenterol 2003; 38: 348-54

7. Yamashita K, Azumano I, Mai M, Okada Y. Expression and tissue localization of matrix metalloproteinase 7 (matrilysin) in human gastric carcinoma. Implication for vessel invasion and metastasis. Int J Cancer 1998; 79: 187-94.

8. Salmela MT, Karjalainen-Lindsberg ML, Puolakkainen P, Saarialho-Kere U. Upregulation and differential expression of matrilysin (MMP-7) and metalloelastase (MMP-12) and their inhibitors TIMP- 1 and TIMP-3 in Barrett's oesophageal adenocarcinoma. BrJ Cancer 2001; 85: 383-92.

9. Tanioka Y, Yoshida T, Ygawa T, et al. Matrix metalloproteinase-7 and matrix metalloproteinase -9 are associated with unfavourable prognosis in superficial oesophageal cancer. BrJ Cancer 2003; 89: 2116-21.

10. Yamamoto $\mathrm{H}$, Adachi $\mathrm{Y}$, Itoh $\mathrm{F}$, et al. Association of matrilysin expression with recurrence and poor prognosis in human esophageal squamous cell carcinoma. Cancer Res 1999; 59: 3313-16.

11. Ishikawa T, Ichikawa Y, Mitsuhashi M, et al. Matrilysin is associated with progression of colorectal tumor. Cancer Lett 1996; 107: 5-10.

12. Masaki T, Sugiyama M, Matsuoka H, et al. Coexpression of matrilysin and laminin-5 $\gamma 2$ chain may contribute to tumor cell migration in colorectal carcinomas. Dig Dis Sci 2003; 48: 1262-67.

13. Kioi M, Yamaoto K, Higashi S, et al. Matrilysin (MMP7) induces homotypic adhesion of human colon cancer cells and enhances their metastatic potential in nude mouse model. Oncogene 2003; $22: 8662-70$.

14. Weinfass B, Nejjari M, Trespeuch C, et al. Effets de la bombésine sur l'expression de la matrilysine MMP7 dans la lignée cancéreuse colique humaine Isreco 1 . Gastroenterol Clin Biol 2004; 28 : 161.

15. Wroblewski LE, Noble PJM, Pagliocca A, et al. Stimulation of MMP-7 (matrilysin) by Helicobacter pylori in human gastric epithelial cells: role in epithelial cell migration. J Cell Sci 2003; 116: 3017-26.

16. Nagashima Y, Hasegawa S, Koshikawa N, et al. Expression of matrilysin in vascular endothelial cells adjacent to matrilysin-producing tumors. Int J Cancer 1997; 72: 441-5.

17. Huo N, Ichikawa Y, Kamiyama M, et al. MMP7 (matrilysin) accelerated growth of human umbilical vein endothelial cells. Cancer Lett 2002; 177: 95-100.

18. Pozzi A, Moberg PE, Miles LA, et al. Elevated matrix metalloprotease and angiostatin levels in integrin alpha 1 knockout mice cause reduced tumor vascularization. Proc Natl Acad Sci USA 2000; 97 : 2202-7.

19. Fingleton B, Vargo-Gogola T, Crawford HC, Matrisian LM. Matrilysin (MMP-7) expression selects for cells with reduced sensitivity to apoptosis. Neoplasia 2001 ; $3: 459-68$.

20. Davies G, Jiang WG, Mason MD. Matrilysin mediates extracellualr cleavage of $\varepsilon$-cadherin from prostate cancer cells: a key mechanism in hepatocyte growth factor/scatter factor-induced cell-cell dissociation and in vitro invasion. Clin Canc Res 2001; 7: 3289-97.
21. McGuire J, Li Q, Parks WC. Matrilysin (matrix metalloproteinase-7) mediates $\varepsilon$-cadherin ectodomain shedding in injured lung epithelium. Am J Pathol 2003; 162: 1831-43.

22. Wilson CL, Matrisian LM. Matrilysin: an epithelial matrix metalloproteinase with potentially novel functions. Int J Biochem Cell Biol 1996; 28: 123-36.

23. Von Bredow DC, Nagle RB, Bowden GT, Cress AE. Cleavage of $\beta 4$ integrin by matrilysin. Exp Cell Res 1997; 236: 341-5

24. Bair EL, Massey CP, Tran NL, et al. Integrin- and cadherin-mediated induction of the matrix metalloprotease matrilysin in cocultures of malignant oral squamous cell carcinoma cells and dermal fibroblasts. Exp Cell Res 2001; 270: 259-67.

25. Leeman MF, Curran S, Murray GI. New insights into the roles of matrix metalloproteinases in colorectal cancer development and progression. Pathol 2003; 201: 528-34.

26. Brabletz $T$, Jung $A$, Dag $S$, et al. $\beta$-catenin regulates the expression of the matrix metalloproteinase-7 in human colorectal cancer. Am J Pathol 1999; 155: 1033-8.

27. Ougolkov AV, Yamashita K, Mai M, Minamoto T. Oncogenic $\beta$-catenin and MMP-7 (matrilysin) cosegregate in late-stage clinical colon cancer. Gastroenterology $2002 ; 122: 60-71$

28. Brabletz T, Jung A, Kirchner T. $\beta$-catenin and the morphogenesis of colorectal cancer. Virchows Arch 2002; 441 : 1-11.

29. Ozaki I, Mizuta $\mathrm{T}$, Yotsumoto $\mathrm{H}$, et al. Involvement of the $E t s-1$ gene in overexpression of matrilysin in human hepatocellular carcinoma. Cancer Res 2000 60: 19-25.

30. Udayakumar TS, Stratton MS, Nagle RB, Bowden GT. Fibroblast growth factor-1 induced promatrilysin expression through the activation of extracellular regulated kinases and STAT3. Neoplasia 2002; $4: 60-7$.

31. Lynch CC, Crawford HC, Matrisian LM, McDonnell S. Epidermal growth factor upregulates matrix metalloproteinase- 7 expression through activation of PEA3 transcription factors. Int J Oncol 2004; 24: 1565-72.

32. Yamamoto $\mathrm{H}$, Iku S, Adachi Y, et al. Association of trypsin expression with tumour progression and matrilysin expression in human colorectal cancer. J Pathol 2003; 199: 176-184.

33. Ueno H, Yamashita K, Azumano I, et al. Enhanced production and activation of matrix metalloproteinase-7 (matrilysin) in human endometrial carcinoma. Int J Cancer 1999; 84: 470-7.

34. Yu WH, Woessner JF Jr. Heparan sulfate proteoglycans as extracellular docking molecules for matrilysin (matrix metalloproteinase 7). J Biol Chem 2000, 275: 4183-91.

35. Shiomi T, Okada Y. MTl-MMP and MMP-7 in invasion and metastasis of human cancers. Cancer Met Rev 2003; 22: 145-52.

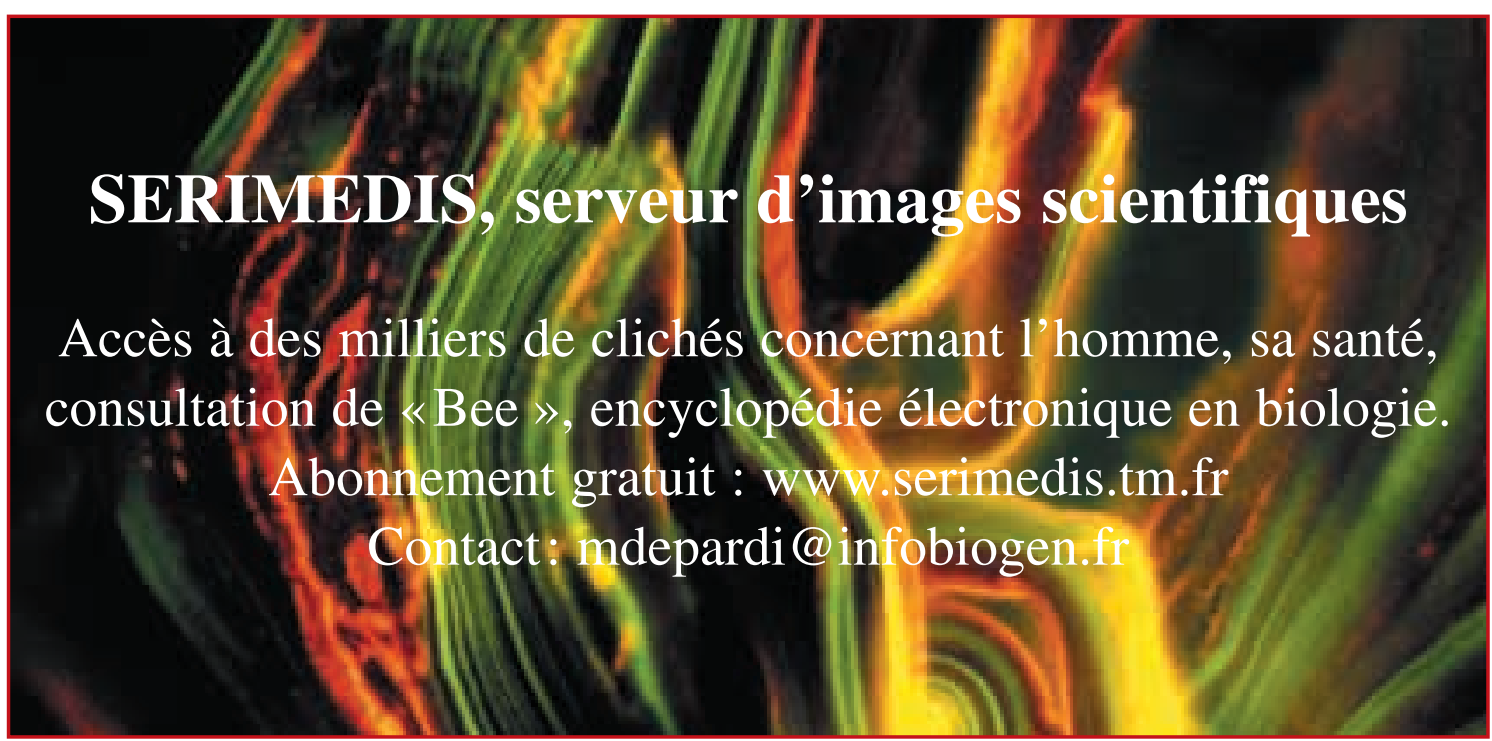

